\title{
Primer registro de Etropus ectenes Jordan, 1889, Bothus constellatus Jordan \& Goss, 1889, Achirus klunzingeri (Steindachner, 1880) y Symphurus elongatus (Günther, 1868) (Pisces, Pleuronectiformes) en Chile, con comentarios sobre la distribución de los lenguados chilenos
}

\author{
Walter Sielfeld ${ }^{1}$, Mauricio Vargas ${ }^{2} \&$ Ismael Kong ${ }^{3}$ \\ ${ }^{1}$ Departamento Ciencias del Mar, Universidad Arturo Prat, Casilla 121, Iquique, Chile \\ ${ }^{2}$ Servicio Nacional de Pesca, I Región, Pasaje Alessandri 470 Of. 110, Iquique, Chile \\ ${ }^{3}$ Facultad de Recursos del Mar, Universidad de Antofagasta, Casilla 170, Antofagasta, Chile \\ E-mail: walter.sielfeld@unap.cl; mvargas@sernapesca.cl; ikong@uantof.cl
}

RESUMEN. Se comunican los primeros registros de los Pleuronectiformes Achirus klunzingeri (Soleidae), Etropus ectenes y Bothus constellatus (Bothidae) y Symphurus elongatus (Cynoglossidae) en aguas de chilenas. Las características morfológicas de los especímenes estudiados se confrontan con descripciones previas. Se discute su distribución en el Pacífico sur oriental y aquella del orden en aguas chilenas.

Palabras claves: Pleuronectiformes, nuevos registros, Etropus ectenes, Bothus constellatus, Achirus klunzingeri, Symphurus elongatus, norte de Chile.

First records of Etropus ectenes Jordan, 1889, Bothus constellatus Jordan \& Goss, 1889, Achirus klunzingeri (Steindachner, 1880) and Symphurus elongatus (Günther, 1868) (Pisces: Pleuronectiformes) in Chile, with comments on the distribution of the Chilean flounders

\begin{abstract}
The first records of the Pleuronectiformes Achirus klunzingeri (Soleidae), Etropus ectenes and Bothus constellatus (Bothidae), and Symphurus elongatus (Cynoglossidae) in chilean waters are reported. The morphological characteristics of the studied specimens are compared with previous descriptions. Their distribution in the Southeastern Pacific and that of the order in Chilean waters are discussed.
\end{abstract}

Key words: Pleuronectiformes, new records, Etropus ectenes, Bothus constellatus, Achirus klunzingeri, Symphurus elongatus, northern Chile.

\section{INTRODUCCIÓN}

Los Pleuronectiformes son peces bénticos y carnívoros, cuya sistemática ha sido discutida ampliamente por Norman (1934), Hubbs (1945), Chabanaud (1949), Amaoka (1969), Li (1981), Hensley \& Ahlstrom (1984), Ahlstrom et al. (1984) y Chapleau (1993).

Las especies chilenas de este orden han sido sintetizadas en los trabajos de Delfín $(1899,1900)$, Fowler (1945), Mann (1954), Bahamonde \& Pequeño (1975), y Pequeño $(1989,1997)$. Aportes puntuales al conocimiento taxonómico de estos peces han sido realizados por Steindachner (1905), Sielfeld (1976), Ojeda (1978), Parin (1983, 1991), Pequeño \& Plaza (1987), Randall \& Meléndez (1987), Hensley \& Suzumoto (1990), Evseenko (1987, 1989, 1990, 1996, 1997, 1998). Incluyendo los nuevos antecedentes que más adelante se presentarán, el orden está representado en aguas chilenas por 28 especies.

Se ha señalado que en Paralichthyidae, Etropus Jordan \& Gilbert, 1882 es componente de un grupo monofilético y se le ha incluido en el grupo 
Cyclopsetta Gill, 1889, junto con Citharichthys Bleeker, 1862 y Syacium Ranzani, 1840, ya que comparten los siguientes caracteres: a) las aletas pélvicas son morfológicamente similares (la base de la aleta pélvica del lado ciego es anterior a la del lado oculado); b) la papila urinaria se sitúa en la misma posición (orientada hacia el lado ciego); c) la aleta caudal posee 17 radios y ninguno de ellos está sostenido por espinas neurales, hemales o preurales), y d) poseen el hipural 5 fusionado con el epural (Hensley \& Ahlstrom, 1984).

La validez de Etropus ha sido mantenida por Hensley \& Ahlstrom (1984), a pesar que es muy similar a Citharichthys, pero no posee la característica protuberancia ósea rostral que tienen muchas especies de Citharichthys.

En el caso de Bothidae se han señalado tres estados derivados para los "bothidos" adultos: a) estado asimétrico de la morfología de la aleta pélvica (alargamiento de la aleta situada en el lado oculado sobre la línea media-ventral del cuerpo); b) pérdida del preorbital del lado ciego; c) presencia de huesos intermusculares (Myorhabdoi) (Hensley \& Ahlstrom, 1984).

En los lenguados achíridos se sigue aquí a Chapleau \& Keast (1988), quienes sobre la base de las características morfológicas del cráneo, han establecido la validez de las familias Soleidae y Achiridae. Se desestima de esta forma la postura tradicional de Soleidae (Soleinae + Achirinae) adoptada por Ahlstrom et al. (1984), Cervigón (1985) y Evseenko (1996).

El presente trabajo, tiene por objeto dar a conocer el registro de cuatro especies de lenguados nuevos para Chile. Se entregan, además, antecedentes morfométricos, merísticos y la distribución geográfica de las especies estudiadas. El estudio se complementa con un análisis de la distribución de los Pleuronectiformes en aguas chilenas.

\section{MATERIALES Y MÉTODOS}

Las medidas y recuentos siguen a Hubbs \& Lagler (1958) con las siguientes excepciones: la altura del cuerpo es la mayor distancia entre la base de los radios dorsales y anales; el grosor corporal correspondió al ancho máximo medido entre la parte ciega y la porción oculada del pez; la longitud preorbital se midió desde el extremo anterior del hocico hasta el borde anterior de la órbita ocular del ojo más inferior; el diámetro ocular comprendió a la distancia anteroposterior del ojo inferior, incluyendo las partes cutáneas anexas; la longitud de los radios dorsales y anales se midieron sobre el lado ciego, y en línea recta desde la base del radio hasta su extremo distal; la longitud de las aletas pélvicas y caudal se midió sobre los radios más largos; el recuento de escamas se realizó en la línea lateral del lado oculado, desde el borde posterior de la cabeza hasta la base de la aleta caudal.

Se utilizaron las siguientes abreviaturas: Lt, longitud total; Ls, longitud estándar; Lc, longitud de la cabeza; Lm, longitud del maxilar; Lh, longitud del hocico; Do, diámetro anteposterior del ojo; Ai, ancho interorbital; Lpo, longitud preorbital; Alcu, altura máxima del cuerpo; Acu, ancho del cuerpo; Alp, altura del pedúnculo caudal; Lpp, longitud prepectoral; Lpd, longitud predorsal; Lpa, longitud preanal; Lpec, longitud de la aleta pectoral; Lpeli, longitud de la aleta pélvica izquierda; Lpeld, longitud de la aleta pélvica derecha; Lac, longitud de la aleta caudal; Lram, longitud radio anal mayor; Lrdm, longitud radio dorsal mayor; Lrpelm, longitud radio pélvico mayor; Lrpecm, longitud radio pectoral mayor. Además se registró el número de radios dorsales (RD), anales (RA), pélvicas (RPel), pectorales $(\mathrm{RPec})$, elementos branquiales (rama superior e inferior del primer arco branquial, incluyendo rudimentos) (BPRS-BPRI); escamas tubulares en la línea lateral (ELL).

La información obtenida, se analizó y comparó con información reportada en publicaciones anteriores sobre las especies en estudio. Sobre la base de información bibliográfica, se resume la información batimétrica y geográfica de las diversas especies de lenguados conocidos para el litoral chileno.

\section{RESULTADOS}

\section{Etropus ectenes Jordan, 1889 (Fig. 1)}

Material examinado

Se examinaron cinco especímenes (116-170 mm Ls) provenientes de una mortandad masiva de peces ocurrida el 5 y 6 de febrero de 1999 en la bahía de Iquique $\left(20^{\circ} 12^{\prime} \mathrm{S}, 70^{\circ} 09^{\prime} \mathrm{W}\right)$, (Walter Sielfeld y Mauricio Vargas colectores). El material se encuentra depositado en la colección zoológica del Departamento de Ciencias del Mar de la Universidad Arturo Prat de Iquique, bajo los números MUAP(PO)-885 a 889. 


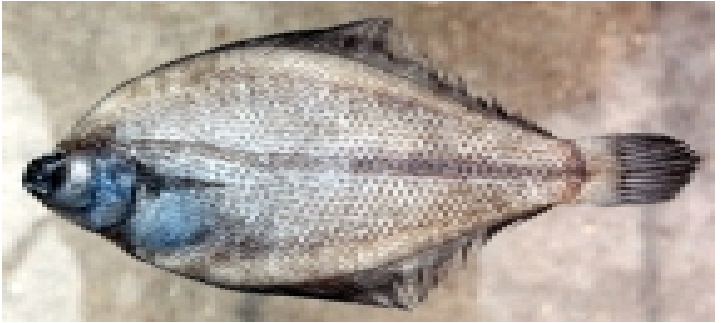

Figura 1. Etropus ectenes MUAP(PO)-886 colectado en bahía de Iquique, Chile (138 mm LS) .

Figure 1. Etropus ectenes MUAP(PO)-886 collected in Iquique bay, (138 mm SL).

\section{Descripción}

Línea dorsal del cuerpo suavemente elevada; cabeza pequeña, 21,2 a 23,3\% de la Ls, su perfil dorsal levemente cóncavo. Alcu entre 43,3 y 45,0\% de la Ls. Ojos muy pequeños y subiguales, separados por una arista ósea; Ai 1,6-2,2\% de la Ls. Boca pequeña, con dientes débiles, dispuestos mayoritariamente en las mandíbulas del lado ciego; el maxilar alcanza la parte anterior del ojo, Lm 2,6-5,5\% de la Ls. RD 88-90 y RA 67-74; aletas pectorales no vestigiales, Rpec 9-10, Rpel 9-10 (Tabla 1); aleta caudal redondeada; ELL 53-56, débilmente ctenoideas en el lado oculado y cicloideas en el lado ciego. Branquispinas pequeñas, en número de 1113 en el primer arco branquial, con 4-6 en la rama superior y 7-9 en la rama inferior. La coloración del lado oculado pardo uniforme y lado ciego no pigmentado.

\section{Distribución geográfica}

Esta especie ha sido citada anteriormente para las costas de Ecuador (Béarez, 1996), el Pacífico colombiano (Rubio, 1988) y Perú (Chirichigno \& Vélez, 1998).

\section{Comentarios}

Los datos merísticos y morfométricos de los especímenes examinados (Tabla 1), concuerdan con los rangos indicados por Hildebrand (1946) para Etropus ectenes Jordan, 1889. Las características señaladas por Norman (1934) para esta especie difieren por el bajo número de RD (59-66 versus 8896), los RA (46-51 versus 67-78) y los Rpec (6 versus 9-12), siendo también menores a los rangos conocidos para Etropus peruvianus Hildebrand, 1946

Tabla 1. Medidas y recuentos de cinco especímenes de Etropus ectenes colectados el 5 y 6 de febrero 1999 en Iquique $\left(2^{\circ} 12^{\prime} \mathrm{S}, 7^{\circ} 09^{\prime} \mathrm{W}\right)$.

Table 1. Measurements and counts of five specimens of Etropus ectenes collected on February $5^{\text {th }}$ and $6^{\text {th }}, 1999$ in Iquique $\left(20^{\circ} 12^{\prime} \mathrm{S}, 7^{\circ} 09^{\prime} \mathrm{W}\right)$.

\begin{tabular}{|c|c|c|c|c|c|c|c|}
\hline \multirow[b]{2}{*}{ Medida (mm) } & \multicolumn{4}{|c|}{ Especimen } & \multirow[b]{2}{*}{5} & \multicolumn{2}{|c|}{ Rango } \\
\hline & 1 & 2 & 3 & 4 & & $\% \mathbf{L t}$ & $\% \mathrm{Ls}$ \\
\hline $\mathrm{Lt}$ & 177,0 & 175,0 & 195,0 & 208,0 & 198,0 & - & - \\
\hline Ls & 140,0 & 138,0 & 157,0 & 170,0 & 116,0 & $58,6-81,7$ & - \\
\hline Lc & 31,0 & 31,0 & 34,0 & 36,0 & 27,0 & $13,6-17,7$ & $21,2-23,3$ \\
\hline $\mathrm{Lm}$ & 6,4 & 7,6 & 8,0 & 8,3 & 3,0 & $1,5-4,3$ & $2,6-5,5$ \\
\hline Do & 6,0 & 7,1 & 8,0 & 8,7 & 6,4 & $3,2-4,2$ & $4,3-5,5$ \\
\hline $\mathrm{Ai}$ & 2,3 & 2,7 & 2,6 & 3,6 & 2,5 & $1,3-1,7$ & $1,6-2,2$ \\
\hline Lpo & 3,7 & 4,0 & 6,0 & 4,4 & 3,8 & $1,9-3,1$ & $2,6-3,8$ \\
\hline Alcu & 63.0 & 61,8 & 69,0 & 73,5 & 51,3 & $25,9-35,6$ & $43,3-45,0$ \\
\hline Alp & 16,4 & 15,9 & 17,3 & 20,0 & 13,0 & $6,6-9,6$ & $11,0-11,8$ \\
\hline Lpec & 22,9 & 22,8 & 25,3 & 24,5 & 19,0 & $9,6-13,0$ & $14,4-16,5$ \\
\hline Lpeli & 9,6 & 12,0 & 14,2 & 13,5 & 11,5 & $5,4-7,3$ & $6,9-9,9$ \\
\hline Lpeld & 13,3 & 14,0 & 16,0 & 17,0 & 13,0 & $6,6-8,2$ & $9,5-11,2$ \\
\hline Datos merísticos & 1 & 2 & 3 & 4 & 5 & \multicolumn{2}{|c|}{ Rango } \\
\hline Rpel & 6 & 6 & 5 & 6 & 5 & \multicolumn{2}{|c|}{$5-6$} \\
\hline $\mathrm{RD}$ & 89 & 88 & 90 & 89 & 88 & \multicolumn{2}{|c|}{$88-90$} \\
\hline RA & 69 & 70 & 74 & 70 & 67 & \multicolumn{2}{|c|}{$67-74$} \\
\hline RPec (lado oculado) & 9 & 10 & 10 & 10 & 9 & \multicolumn{2}{|c|}{$9-10$} \\
\hline BPRS-BPRI & $5+8$ & $4+7$ & $6+8$ & $4+8$ & $4+9$ & \multicolumn{2}{|c|}{$4-6+7-9$} \\
\hline
\end{tabular}


(Tabla 2), especie que es simpátrica con E. ectenes en las costas de Perú. La identidad del material estudiado por Norman (1934) requiere en consecuencia de una revisión posterior y que escapa de los objetivos del presente trabajo.

\section{Bothus constellatus Jordan \& Goss, 1889}

(Fig. 2)

\section{Material examinado}

Un espécimen de Lt 132,8 mm, colectado el 15 de noviembre 1998 en Caleta Buena (19 $53^{\circ} \mathrm{S}, 70^{\circ}$ 08'W), Gustavo Ceballos colector. El material ha sido depositado en la colección zoológica del Departamento de Ciencias del Mar de la Universidad Arturo Prat de Iquique, bajo el número MUAP(PO)890.

\section{Descripción}

La Lc 26,1\% de la Ls y Alcu 65,2\% de la misma; ojos grandes, ampliamente separados, ubicados en el lado izquierdo del cuerpo, Do igual a 5\% de la Ls y contenido 2,4 veces en Ai; el borde anterior del ojo superior aproximadamente a nivel del centro del ojo inferior. Con una espina rostral roma y sin espinas orbitales. Lm igual a 21,4\% de Lc (Tabla 3). Preopérculo con el margen libre, no recubierto por la piel. Aletas pectorales desarrolladas, Lpec del lado oculado levemente menor a Lc, con 10 radios. Inicio de la aleta dorsal adelantada, a nivel del extre-

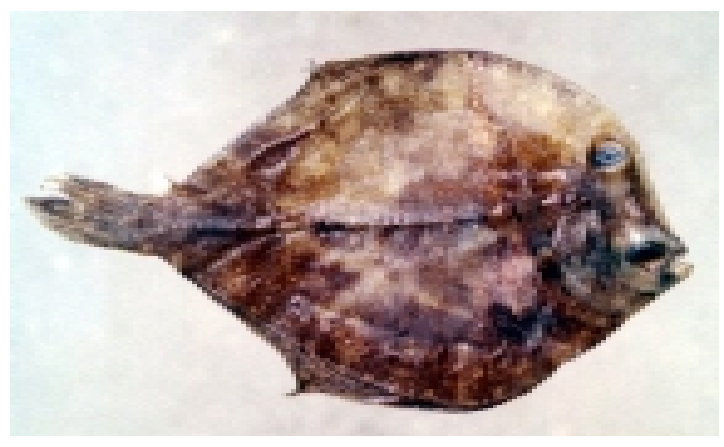

Figura 2. Bothus constellatus MUAP(PO)-890 collected in Caleta Buena (111 mm LS).

Figure 2. Bothus constellatus MUAP(PO)-890 collected in Caleta Buena (111 mm SL)).

mo posterior del maxilar; RD 89, RA 64; RPel del lado oculado 3; Rpel del lado ciego 5; ELL 72, conspicua y fuertemente recurvada en su inicio a nivel del origen de la aleta pectoral; la porción restante recta. Escamas en general pequeñas, que cubren cabeza, cuerpo y radios. Las escamas del lado oculado son ctenoídeas, las del lado ciego cicloideas. BPRS-BPRI 7+9 (Tabla 4); branquispinas pequeñas y cortas; coloración dorsal parda uniforme y ventral no pigmentada.

\section{Distribución geográfica}

Bahía de Panamá e Islas Galápagos (Norman, 1934), Baja California a Perú e Islas Galápagos

Tabla 2. Datos comparativos de Etropus ectenes obtenidos en este estudio (*), con los registros de Norman (1934) (**) y Hildebrand (1946) (***). Se agregan los caracteres distintivos de Etropus peruvianus Hildebrand, 1946 (****) según Hildebrand (1946).

Table 2. Comparative data of Etropus ectenes obtained during this study (*), with records of Norman (1934) (**) and Hildebrand (1946) (***). Distinguishing characters of Etropus peruvianus Hildebrand, $1946(* * * *)$ taken from Hildebrand (1946) are added.

\begin{tabular}{|lcccc|}
\hline Medidas y recuentos & $*$ & $* *$ & $* * *$ & $* * * *$ \\
\hline Ls (mm) & $116-170$ & $75-90$ & $50-200$ & $42-65$ \\
Lc en Ls (veces) & $4,30-4,72$ & 4,0 & $4,1-4,8$ & $3,4-4,0$ \\
Alcu en Ls (veces) & $2,22-2,31$ & $2,25-2,6$ & $2,1-2,6$ & $2,5-2,9$ \\
Lpec en Lc (veces) & $1,34-1,47$ & - & $1,3-1,5$ & 1,25 \\
RD & $88-90$ & $59-66$ & $89-96$ & $82-86$ \\
RA & $67-74$ & $46-51$ & $68-78$ & $64-65$ \\
Rpel & $5-6$ & - & - & - \\
Rpec lado oculado & $9-10$ & 6 & $10-12$ & 10 \\
BPRS-BPRI & $4-6+7-9$ & - & - & - \\
\hline
\end{tabular}


Tabla 3. Antecedentes morfométricos y merísticos de Bothus constellatus colectado el 15 de noviembre 1998 en Caleta Buena, Chile (19 53 'S, 70 ${ }^{\circ} 08^{\prime}$ 'W).

Table 3. Measurements and counts of Bothus constellatus collected on November $15^{\text {th }} 1998$ in Caleta Buena, Chile (1953'S, $\left.7^{\circ} 08^{\prime} \mathrm{W}\right)$.

\begin{tabular}{|c|c|c|c|}
\hline Medida & $\mathbf{m m}$ & $\% \mathbf{L t}$ & $\% \mathrm{Ls}$ \\
\hline $\mathrm{Lt}$ & 132,8 & - & - \\
\hline Ls & 111,0 & 83,6 & - \\
\hline Lc & 29,0 & 21,8 & 26,1 \\
\hline $\mathrm{Lm}$ & 6,2 & 4,7 & 5,6 \\
\hline Do & 5,6 & 4,2 & 5,0 \\
\hline $\mathrm{Lh}$ & 5,5 & 4,1 & 5,0 \\
\hline $\mathrm{Ai}$ & 13,7 & 10,3 & 12,3 \\
\hline Alcu & 72,4 & 54,5 & 65,2 \\
\hline Alp & 6,3 & 4,7 & 5,7 \\
\hline Lpec & 21,8 & 16,4 & 19,6 \\
\hline Lpd & 3,7 & 2,8 & 3,3 \\
\hline Lpp & 27,0 & 20,3 & 24,3 \\
\hline Lpa & 32,7 & 24,6 & 29,5 \\
\hline Lac & 22,5 & 16,9 & 20,3 \\
\hline Lram & 10,8 & 8,1 & 9,7 \\
\hline Lrdm & 10,6 & 8,0 & 9,5 \\
\hline \multicolumn{4}{|c|}{ Datos merísticos } \\
\hline \multicolumn{2}{|c|}{ Rpel lado ciego } & \multicolumn{2}{|l|}{5} \\
\hline \multicolumn{2}{|c|}{ Rpel lado oculado } & \multicolumn{2}{|l|}{3} \\
\hline \multicolumn{2}{|l|}{$\mathrm{RD}$} & \multicolumn{2}{|l|}{89} \\
\hline \multicolumn{2}{|l|}{ RA } & \multicolumn{2}{|l|}{64} \\
\hline \multicolumn{2}{|l|}{ Rpec } & \multicolumn{2}{|l|}{10} \\
\hline \multicolumn{2}{|c|}{ BPRS-BPRI } & \multicolumn{2}{|l|}{$9+7$} \\
\hline
\end{tabular}

(Hildebrand, 1946), costa continental de Ecuador (Béarez, 1996) y Pacífico colombiano (Rubio, 1988).

\section{Comentarios}

Las características del espécimen estudiado corresponden con los detalles entregados por Norman (1934) y Hildebrand (1946), al igual que las características resumidas en la Tabla 4. Esta especie difiere de Bothus mancus (Broussonet, 1782) por el menor número de escamas en la línea lateral, elementos dorsales y anales (Tabla 5).

\section{Achirus klunzingeri (Steindachner, 1880)}

(Fig. 3)

\section{Material examinado}

Un espécimen de $238 \mathrm{~mm}$ de Lt capturado el 24 de marzo de 1984 en la bahía de Mejillones (23º5' $\mathrm{S}$, $\left.70^{\circ} 28^{\prime} \mathrm{W}\right)$, Ismael Kong colector, depositado en la colección zoológica del Departamento de Ciencias del Mar de la Universidad Arturo Prat de Iquique, bajo el número MUAP(PO)-891.

\section{Descripción}

Cuerpo ovalado, Alcu igual a 67,7\% de Ls. La cabeza es escamada, excepto en el área peribucal y las membranas branquiostegales; septo interbranquial atravesado por un foramen que comunica ambas cámaras branquiales. Lc igual a 23,7\% de Ls; ojos pequeños, y subiguales, situados al lado derecho de la cabeza. Do contenido 19,6 veces en Ai, e igual a $1,5 \%$ de la Ls; boca pequeña con labios carnosos

Tabla 4. Datos comparativos de Bothus constellatus obtenidos en este estudio (*), comparados con los registros de Norman, $1934(* *)$, Hildebrand, $1946(* * *)$ y Chirichigno \& Vélez, 1998 (****).

Table 4. Comparative data of Bothus constellatus obtained during this study (*), compared with records of Norman, $1934(* *)$ and Hildebrand, 1946 (***) and Chirichigno \& Vélez, 1998 (****).

\begin{tabular}{|lcccc|}
\hline Medida & $*$ & $* *$ & $* * *$ & $* * * *$ \\
\hline Ls (mm) & 111 & - & $52-78$ & - \\
Lc en Ls (veces) & 3,8 & 4,0 & $3,5-4,0$ & - \\
Alcu en Ls (veces) & 1,53 & $1,5-1,75$ & $1,4-1,7$ & - \\
Lpec en Lc (veces) & 1,03 & 1,25 & - & - \\
RD & 89 & 89 & $85-90$ & - \\
RA & 56 & 65 & $62-68$ & 65 \\
Rpec & 10 & 12 & $10-12$ & - \\
ELL & 72 & 75 & $72-79$ & - \\
BPRS-BPRI & $7+9$ & - & $5-7+9-11$ & - \\
& & & & \\
\hline
\end{tabular}


Tabla 5. Resumen de los principales caracteres distintivos entre las especies chilenas del género Bothus Rafinesque, 1810 basado en Norman, 1934 (*), De Buen, 1959 (**) y este trabajo (***).

Table 5. Principal distinctive characters between Chilean species of genus Bothus Rafinesque, 1810 based on Norman, $1934(*)$, De Buen, 1959 (**) and present results (***).

\begin{tabular}{|lcc|}
\hline Especie & B. mancus & B. constellatus $(* * *)$ \\
\hline Alcu en Ls (veces) & $1,7(* *)$ & $1,40-1,75$ \\
Lc en Ls (veces) & $3,7-4,0(* *)$ & $3,5-4,0$ \\
Lpec del macho en Lc (veces) & $0,4(*)$ & $1,03-1,25$ \\
Do en Lc (veces) & $4,66-6,5(*)$ & $4,0-4,1$ \\
RD & $98-103(*)$ & $85-90$ \\
RA & $76-80(*)$ & $62-68$ \\
Rpec & $11-12(* *)$ & $10-12$ \\
ELL & $85-90(*)$ & $72-79$ \\
BPRS-BPRI & $9-11(*)$ & $9-11$ \\
\hline
\end{tabular}

Tabla 6. Medidas y recuentos de Achirus klunzingeri colectado el 24 de marzo 1984 en la bahía de Mejillones $\left(2^{\circ} 05 ' S, 7^{\circ} 28^{\prime} \mathrm{W}\right)$,

Table 6. Measurements and counts of Achirus klunzingeri collected on March $24^{\text {th }} 1984$ in bay of Mejillones (23'05'S, 70 $\left.^{\circ} 28^{\prime} \mathrm{W}\right)$.

\begin{tabular}{|c|c|c|c|}
\hline Medida & $\mathbf{m m}$ & $\% \mathbf{L t}$ & $\% \mathrm{Ls}$ \\
\hline $\mathrm{Lt}$ & 238,0 & - & - \\
\hline Ls & 198,0 & 83,2 & - \\
\hline Lc & 47,0 & 19,7 & 23,7 \\
\hline $\mathrm{Lm}$ & 11,0 & 4,6 & 5,6 \\
\hline Do & 3,0 & 1,3 & 1,5 \\
\hline $\mathrm{Ai}$ & 8,8 & 3,7 & 4,4 \\
\hline $\mathrm{Lh}$ & 14,0 & 5,9 & 7,1 \\
\hline Acu & 23,0 & 9,7 & 11,6 \\
\hline Alcu & 134,0 & 56,3 & 67,7 \\
\hline Alp & 32,0 & 13,4 & 16,2 \\
\hline Lpec lado oculad & do 2,4 & 1,0 & 1,2 \\
\hline Lpd & 9,0 & 3,8 & 4,5 \\
\hline Lpa & 61,5 & 25,8 & 31,1 \\
\hline $\mathrm{Lac}$ & 39,2 & 16,5 & 19,8 \\
\hline Lram & 16,0 & 6,7 & 8,1 \\
\hline Lrpecm & 2,4 & 1,0 & 1,2 \\
\hline Lrpelm & 8,2 & 3,4 & 4,1 \\
\hline Lrdm & 16,0 & 6,7 & 8,1 \\
\hline \multicolumn{4}{|c|}{ Datos merísticos } \\
\hline Rpel & & 4 & \\
\hline $\mathrm{RD}$ & & 61 & \\
\hline RA & & 46 & \\
\hline Rpec lado oculad & & 2 & \\
\hline BPRS-BPRI & & $5+5$ & \\
\hline
\end{tabular}

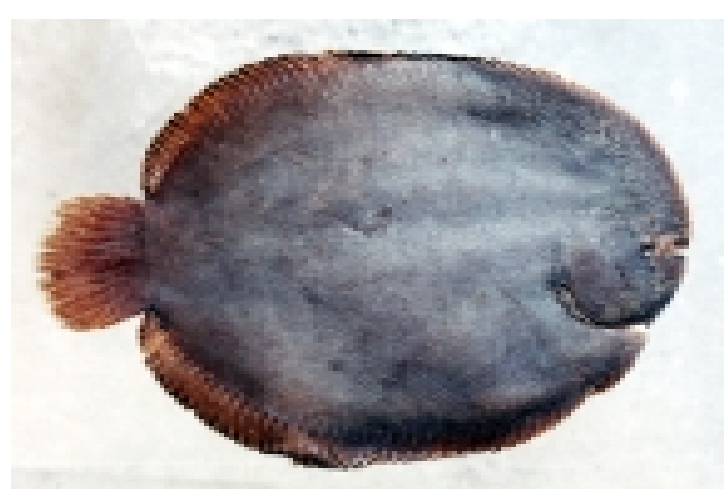

Figura 3. Achirus klunzingeri MUAP(PO)-891 colectado en bahía de Mejillones (198 mm LS).

Figure 3. Achirus klunzingeri MUAP(PO)-891 collected in Mejillones bay (198 mm SL).

provistos de circunvoluciones dérmicas con dientes pequeños y poco aparentes. El proceso anterior del maxilar es liso y no se prolonga como espina. Borde preopercular no libre y poco aparente. En la Tabla 6 se presentan las características morfométricas y merísticas del ejemplar estudiado. RD 61; la aleta dorsal se inicia por delante de los ojos, sobre el hocico (a $9 \mathrm{~mm}$ del borde anterior del labio superior); los radios anteriores de la aleta dorsal están recubiertos por la piel; la aleta pectoral del lado oculado sumamente corta, RPec 2; no se aprecia aleta en el lado ciego; RA 46. La aleta caudal es libre y redondeada, libre de la aleta dorsal y anal; los radios están recubiertos por escamas. Aletas pélvicas con la base alargada; la del lado oculado se fusiona con la aleta anal y presenta 4 radios más cortos que 
la base de la aleta; los radios recubiertos por escamas. Línea lateral no aparente; en una serie longitudinal desde el opérculo a la aleta caudal 58 filas de escamas. Las escamas del lado oculado son fuertemente ctenoideas, pequeñas y cubren el cuerpo, cabeza, base de las aletas y $3 / 4$ basales de los radios dorsales, anales y caudales. BPRS-BPRI 5+5; todas las branquispinas cortas y redondeadas las de la rama inferior dispuestas en su tercio superior. Coloración del lado oculado parda uniforme, lado ciego de coloración blanquecina.

\section{Distribución geográfica}

Esta especie ha sido citada anteriormente en Isla de Pascua (Pequeño, 1989), cita que requiere de confirmación ya que Hensley \& Suzumoto (1990) no incluyen a esta especie en su revisión de ese sector insular. Béarez (1996) señalan las costas de Ecuador y Rubio (1988) el Pacífico colombiano.

El presente espécimen permite extender la distribución de esta especie hasta la zona de Iquique en el norte de Chile $\left(20^{\circ} \mathrm{S}\right)$. La presencia de esta especie en esta latitud tiene relación con el evento cálido de El Niño 1982/83, entre cuyas consecuencias se ha indicado la migración hacia el sur de diversas especies de peces correspondientes a la zooprovincia peruana (Kong et al., 1985).

\section{Comentarios}

Según Tabla 7, el número de elementos dorsales, ventrales y anales del presente ejemplar, concuerdan con los rangos que indican Chirichigno \& Vélez (1998), y Rubio (1988) para esta especie. Las aletas

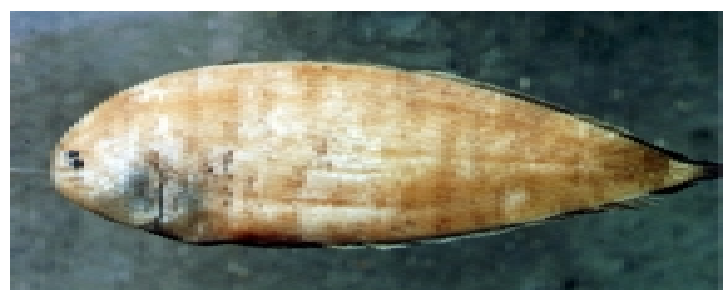

Figura 4. Symphurus elongatus MUAP(PO)-892 colectado en Arica (162 mm LS).

Figure 4. Symphurus elongatus MUAP(PO)-892 collected in Arica (162 mm SL).

pectorales son vestigiales y faltan en el lado ciego. La pectoral del lado oculado de este espécimen solo presenta 2 radios, aspecto que contrasta con 4-5 radios vestigiales indicados por otros autores. No obstante, esta variabilidad también es señalada para otras especies del género. Así, Cervigón (1985) señala para $A$. achirus (Linnaeus, 1758) que su aleta pectoral del lado oculado presentaría entre 0-5 radios, A. lineatus (Linnaeus, 1758) 3-8 radios y $A$. novoae Cervigón, 1982 2-4 radios.

\section{Symphurus elongatus (Günther, 1868) (Fig. 4)}

\section{Material examinado}

Un espécimen de $171 \mathrm{~mm}$ de Lt, capturado el 14 de noviembre 2000 en Arica (18²8'S, 70¹9'W), Carlos Gaspar colector y depositado en la colección zoológica del Departamento de Ciencias del Mar de la Universidad Arturo Prat de Iquique, bajo el número MUAP(PO)-892.

Tabla 7. Datos comparativos de Achirus klunzingeri obtenidos en este estudio (*), comparado con los registros de Chirichigno \& Vélez (1998) (**) y Rubio (1988) (***).

Table 7. Comparative data of Achirus klunzingeri obtained from the present study (*), compared with those of Chirichigno \& Vélez (1998) (**) and Rubio (1988) (***).

\begin{tabular}{|lccc|}
\hline Medida & $*$ & $* *$ & $* * *$ \\
\hline Ls (mm) & 198 & - & - \\
Lc en Ls (veces) & 4,2 & - & - \\
Alcu en Ls (veces) & 1,5 & - & - \\
Lpec en Lc (veces) & 19,6 & $59-66$ & $59-66$ \\
RD & 61 & $46-51$ & $46-51$ \\
RA & 46 & - & - \\
Rpel & 4 & 6 & $5-6$ \\
RPec lado oculado & 2 & - & \\
\hline
\end{tabular}




\section{Descripción}

Cuerpo alargado, Alcu igual a 26,0\% de Ls; Lc representa 14,2\% de Ls (Tabla 8). Los ojos pequeños, Do del ojo inferior igual a 10,0\% de Lc; sin opérculo pupilar. Interorbita pequeña, igual a 30,4\% de Do. Hocico redondeado, no sobrepasando por delante del maxilar. El maxilar sobrepasa el borde posterior de la pupila del ojo inferior y alcanza la órbita posterior del mismo. La aleta dorsal larga, comienza al nivel de la órbita anterior del ojo superior que es el más adelantado; Lrdm igual a 1,3\% de Lt; Lram igual a 4,6\% de Ls. Ambas aletas sin escamas sobre los radios. Solo con una aleta ventral, la que tiene disposición en la línea ventral media; no se une con la anal; el orificio anal está desplazado hacia el lado ciego. RD 108, RA 89; aleta pélvica solo presente en el lado izquierdo; RPel 4; Lrpelm igual a 4,6\%

Tabla 8. Medidas y recuentos de Symphurus elongatus (Günther, 1868) colectado el 14 de noviembre 2000 en Arica (18 28 'S, $7^{\circ} 19^{\prime} W$ ).

Table 8. Measurements and counts of Symphurus elongatus (Günther, 1868) collected on november $14^{\text {th }}$ 2000 in Arica (18 $\left.28^{\prime} \mathrm{S}, 7^{\circ} 19^{\prime} \mathrm{W}\right)$.

\begin{tabular}{|c|c|c|c|}
\hline Medida & $\mathbf{m m}$ & $\% \mathbf{L t}$ & $\% \mathrm{Ls}$ \\
\hline $\mathrm{Lt}$ & 171,0 & - & - \\
\hline Ls & 162,0 & 94,7 & - \\
\hline $\mathrm{Lc}$ & 23,0 & 13,5 & 14,2 \\
\hline $\mathrm{Lm}$ & 10,9 & 6,4 & 6,7 \\
\hline Do & 2,3 & 1,3 & 1,4 \\
\hline $\mathrm{Ai}$ & 0,8 & 0,5 & 0,5 \\
\hline $\mathrm{Lh}$ & 11,8 & 7,0 & 7,3 \\
\hline Acu & 10,1 & 6,3 & 6,2 \\
\hline Alcu & 44,4 & 26,0 & 27,4 \\
\hline Alp & 3,9 & 2,3 & 2,4 \\
\hline Lpd & 10,1 & 6,0 & 6,2 \\
\hline Lpa & 29,8 & 17,4 & 18,4 \\
\hline $\mathrm{Lac}$ & 9,0 & 5,3 & 5,6 \\
\hline Lram & 7,5 & 4,4 & 4,6 \\
\hline Lrpelm & 8,9 & 5,2 & 5,5 \\
\hline $\mathrm{Ldm}$ & 7,4 & 4,3 & 4,6 \\
\hline \multicolumn{4}{|c|}{ Datos merísticos } \\
\hline \multicolumn{2}{|c|}{ RPel (solo lado izquierdo) } & \multicolumn{2}{|l|}{4} \\
\hline \multicolumn{2}{|l|}{ RD } & 107 & \\
\hline \multicolumn{2}{|l|}{ RA } & 89 & \\
\hline \multicolumn{2}{|c|}{ Escamas serie longitudinal } & 92 & \\
\hline
\end{tabular}

de Ls. Escamas de ambos lados ctenoideas; en una línea lateral desde el ángulo del opérculo a la aleta caudal, 97 filas oblicuas de escamas en el lado ciego y 94 filas oblicuas en el lado oculado. Los radios de las aletas dorsal y anal no están cubiertos por escamas. Primer arco branquial sin branquispinas aparentes. La rama superior con el borde recto. BPRI con 15 pequeñas tuberosidades desiguales, que solamente comprometen la mucosa, y están dispuestas irregularmente en el tercio más superior de la rama. Lado ciego de color blanco, salvo la aleta caudal y la parte posterior de las aletas anal y dorsal que son negras; lado oculado de color pardo muy claro, destacando alrededor de 16 bandas transversales angostas, muy difusas y distribuidas sobre los 3/4 anteriores del cuerpo; tanto la aleta anal como la dorsal pigmentadas con pequeños puntos, más abundantes sobre los radios; aleta caudal negra al igual que la parte posterior de las aletas dorsal y anal; el peritoneo sin ser negro, se trasluce al exterior.

\section{Distribución geográfica}

Esta especie ha sido anteriormente señalada para el litoral de Ecuador (Massay, 1983) y golfo de Panamá (Hildebrand, 1946). Chirichigno \& Vélez (1998) sintetizan estas citas con datos de Perú e indican como área distribucional el litoral Pacífico oriental entre Centroamérica y Huacho $\left(11^{\circ} \mathrm{S}\right)$. Munroe et al. (1995) esquematizan el área de distribución entre $\operatorname{los} 25^{\circ} \mathrm{N}$ frente a Baja California y alrededor de $\operatorname{los} 2^{\circ} \mathrm{S}$ frente a Ecuador. En consecuencia, el presente material extiende el rango distribucional conocido de la especie en alrededor de siete y medio grados geográficos al sur y constituye la primera cita de la familia Cynoglossidae para la costa de Chile.

\section{Comentarios}

Algunos datos morfométricos y merísticos de los peces de este estudio, y su comparación con las especies mas próximas, muestran clara correspondencia con S. elongatus, especie con la cual el presente material comparte también otras características como son la ausencia de franjas obscuras nítidas y, si existen franjas difusas, están siempre en número mayor a cuatro (Tablas 9 y 10). El presente ejemplar, muestra más de diez franjas muy difusas. Es también característico de la especie, la ausencia de pigmentos en el peritoneo, aún cuando se traslucen parcialmente las vísceras más obscuras. 
Tabla 9. Comparación entre diversas especies de Symphurus de maxilar largo (tomado de Hildebrand, 1946) y el presente ejemplar $(*)$.

Table 9. Comparison among the present specimen (*) and other species with long maxillary of the genus Symphurus (after Hildebrand, 1946).

\begin{tabular}{|lcccc|}
\hline Medida & $*$ & S. elongatus & S. leei & S. sechurae \\
\hline Lt (mm) & 171 & - & 14,8 & - \\
Lc (\% de Ls) & 14,2 & - & - & - \\
Alcu (\% de Ls) & 27,4 & - & - & - \\
RD & 107 & $99-107$ & $93-104$ & - \\
RA & 89 & $83-90$ & $77-88$ & - \\
Rpel & 4 & & - & - \\
Escamas en & & & & \\
serie longitudinal & 91 & $87-92$ & $82-87$ & $84-89$ \\
\hline
\end{tabular}

Tabla 10. Datos comparativos de Symphurus elongatus (Günther, 1868) obtenidos en este estudio (*), comparados con los registros de Hildebrand (1946)(**), Chirichigno \& Vélez (1998)(***) y Munroe et al. (1995)(***).

Table 10. Comparative data of Symphurus elongatus (Günther, 1868) obtained from the present study (*), compared with the records of Hildebrand (1946)(**), Chirichigno \& Vélez (1998)(***) and Munroe et al. (1995)(***).

\begin{tabular}{|lccc|}
\hline Medida & $*$ & $* *$ & $* * *$ \\
\hline Lt (mm) & 171 & - & 15,8 \\
Lc (\% de Ls) & 14,2 & - & - \\
Alcu (\% de Ls) & 27,4 & $22,2-23,3$ & - \\
RD & 107 & $100-105$ & $99-107$ \\
RA & 89 & $83-86$ & $83-90$ \\
Rpel & 4 & - & - \\
Escamas en & & & \\
serie longitudinal & 91 & $87-92$ & - \\
\hline
\end{tabular}

\section{DISCUSIÓN}

Sobre la base de las presentes nuevas adiciones, se reconocen 28 especies de lenguados en el litoral chileno, repartidas en 16 géneros y 5 familias (Tabla 11), ordenadas según Nelson (1994), a excepción de la familia Achiropsettidae cuya validez como familia independiente ha sido propuesta por Evseenko (1984). En la Tabla 12 se resumen datos sobre distribución batimétrica, distribución geográfica y las fuentes bibliográficas respectivas.

La familia Bothidae destaca como la más numerosa, con 21 especies, donde Scopthalminae (género Thysanopsetta Günther, 1880) tiene una especie, Paralichthyinae (géneros
Hippoglossina Steindachner, 1876, Paralichthys Girard, 1858 y Etropus Jordan \& Gilbert, 1882) reúne 12 especies y Bothinae (géneros Arnoglossus Bleeker, 1862, Pelecanichthys Gilbert \& Cramer, 1897, Chascanopsetta Alcock, 1894, Bothus Rafinesque, 1810, Parabothus Norman, 1931 y Engyprosopon Günther, 1862) reúne 8 especies.

La familia Archiropsettidae (géneros Achiropsetta Norman, 1930, Mancopsetta Gill, 1881 y Pseudomancopsetta Evseenko, 1984) incluye 4 especies, y Achiridae (género Achirus Lacépede, 1802), Cynoglossidae (género Symphurus Rafinesque, 1810) y Soleidae (género Aseraggodes Kaup, 1858) están representadas cada una por una especie.

El género Thysanopsetta (Bothidae, Scopthalminae) es monoespecífico, y se distribuye en profundidades de 90-100 m en el Atlántico austral, Malvinas y Pacifico austral desde los 42 a $53^{\circ} \mathrm{S}$ (Norman, 1934).

De la subfamilia Bothinae, las especies de Arnoglossus habitan aguas con profundidades de 150-700 m, en el Indopacífico, el Mediterráneo, y la costa Atlántica de Africa y Europa. Los géneros Parabothus y Chascanopsetta son exclusivos del Indopacífico (Norman, 1934) y Pelecanichthys es propio del océano Indico y del Pacífico en Hawaii y Cordillera de Nazca (Amaoka \& Parin, 1990).

El género Engyprosopon habita el litoral somero con profundidades de 0-10 $\mathrm{m}$ en islas del Pacífico tropical (Norman, 1934; Hensley \& Suzumoto, 1990). Los representantes del género Bothus se distribuyen en aguas cálidas del Atlántico, Mediterráneo e Indopacífico (Norman, 1934), incluyendo el litoral de isla de Pascua y el continente sudamericano.

En Paralichthyinae el género Hipoglossina se distribuye en el Pacifico oriental desde California a Chile, Paralichthys en las costas de China, Japón, la costa Pacífica hasta Patagonia, y por la costa Atlántica desde Patagonia hasta Nueva York, y Etropus en ambas costas de América, donde las especies pacíficas son tropicales y subtropicales (Norman, 1934; 
Ginsburg, 1952).

En la familia Cynoglossidae, las especies del género Symphurus habitan en su mayoría, el litoral somero Atlántico tropical y subtropical de Macronesia, Africa, Mar Mediterráneo, y el Caribe hasta Argentina (Munroe et al., 2000). En el Pacífico cuatro especies se distribuyen entre Baja California y el norte de Chile (Munroe, 1992).

El género Aseraggodes (Soleidae) habita a profundidades de 1-25 m en el litoral de isla de Pascua y Lord Howe (Randall \& Meléndez, 1987).

El género Achirus (Achiridae) es propio del litoral somero de la costa pacífica desde la península de Mazatlán hasta el norte de Chile (Krupp, 1995 y este estudio), además del sector tropical y subtropical de la costa Atlántica (Paiva Carvalho et al., 1968; De Buen, 1950; Cervigón, 1985). Incluye algunas especies exclusivas de aguas salobres e incluso dul- ces (Cervigón, 1985).

Finalmente, Achiropsettidae es de distribución austral (al sur $44^{\circ} \mathrm{S}$ ), con especies que habitan aguas más bien profundas de 100-1000 m, donde Achiropsetta habita en aguas antárticas y subantárticas (Heemstra, 1990), Mancopsetta en los océanos Atlántico, Pacífico e Indico australes (Evseenko,1997), y Pseudomancopsetta, que es monoespecífico, en el Pacífico austral (Evseenko, 1984).

En el conjunto de especies chilenas destacan por lo tanto cuatro grupos de especies de lenguados, que derivan de unidades zoogeográficas distintas. Así, el litoral chileno central y sur, incluyendo las islas del archipiélago de Juan Fernández, se caracteriza por la presencia de Bothidae Paralichthyinae (géneros Hippoglossina y Paralichthys). Sus especies han invadido lagunas salobres costeras (Paralichthys

Tabla 11. Ordenación taxonómica de los lenguados de aguas chilenas.

Table 11. Taxonomic arrangement of Chilean flatfishes.

\begin{tabular}{|c|c|}
\hline Familia Achiropsettidae & $\begin{array}{l}\text { Achiropsetta tricholepis Norman, } 1930 \\
\text { Mancopsetta maculata (Günther, 1880) } \\
\text { Mancopsetta milfordi Penrith, 1965 } \\
\text { Pseudomancopsetta andriashevi Evseenko, } 1984\end{array}$ \\
\hline Familia Bothidae & \\
\hline Subfamilia Scophthalminae & Thysanopsetta naresi Günther, 1880 \\
\hline Subfamilia Bothinae & $\begin{array}{l}\text { Arnoglossus multirastris Parin, } 1983 \\
\text { Pelecanichthys crumenalis Gilbert \& Cramer, } 1897 \\
\text { Chascanopsetta megagnatha Amaoka \& Parin, } 1990 \\
\text { Bothus mancus (Broussonet, 1782) } \\
\text { Bothus constellatus (Jordan, 1889) } \\
\text { Parabothus amaokai Parin, 1983 } \\
\text { Engyprosopon arenicola Jordan \& Evermann, } 1903 \\
\text { Engyprosopon regani Hensley \& Suzumoto, } 1990\end{array}$ \\
\hline Subfamilia Paralichthyinae & $\begin{array}{l}\text { Hippoglossina macrops Steindachner, } 1876 \\
\text { Hippoglossina mystacium Ginsburg, } 1936 \\
\text { Hippoglossina montemaris de Buen, } 1961 \\
\text { Paralichthys adpersus (Steindachner, 1867) } \\
\text { Paralichthys coeruleosticta Steindachner, } 1898 \\
\text { Paralichthys delfini Pequeño \& Plaza, } 1987 \\
\text { Paralichthys fernadezianus Steindachner, } 1903 \\
\text { Paralichthys hilgendorfi Steindachner, } 1903 \\
\text { Paralichthys microps (Günther, 1881) } \\
\text { Paralichthys patagonicus Jordan, 1889 } \\
\text { Paralichthys schmitti Ginsburg, } 1933 \\
\text { Etropus ectenes Jordan, } 1889\end{array}$ \\
\hline Familia Achiridae & Achirus klunzingeri (Steindachner, 1880) \\
\hline Familia Soleidae & Aseraggodes bahamondei Randall \& Meléndez, 1987 \\
\hline Familia Cynoglossidae & Symphurus elongatus (Günther, 1868) \\
\hline
\end{tabular}




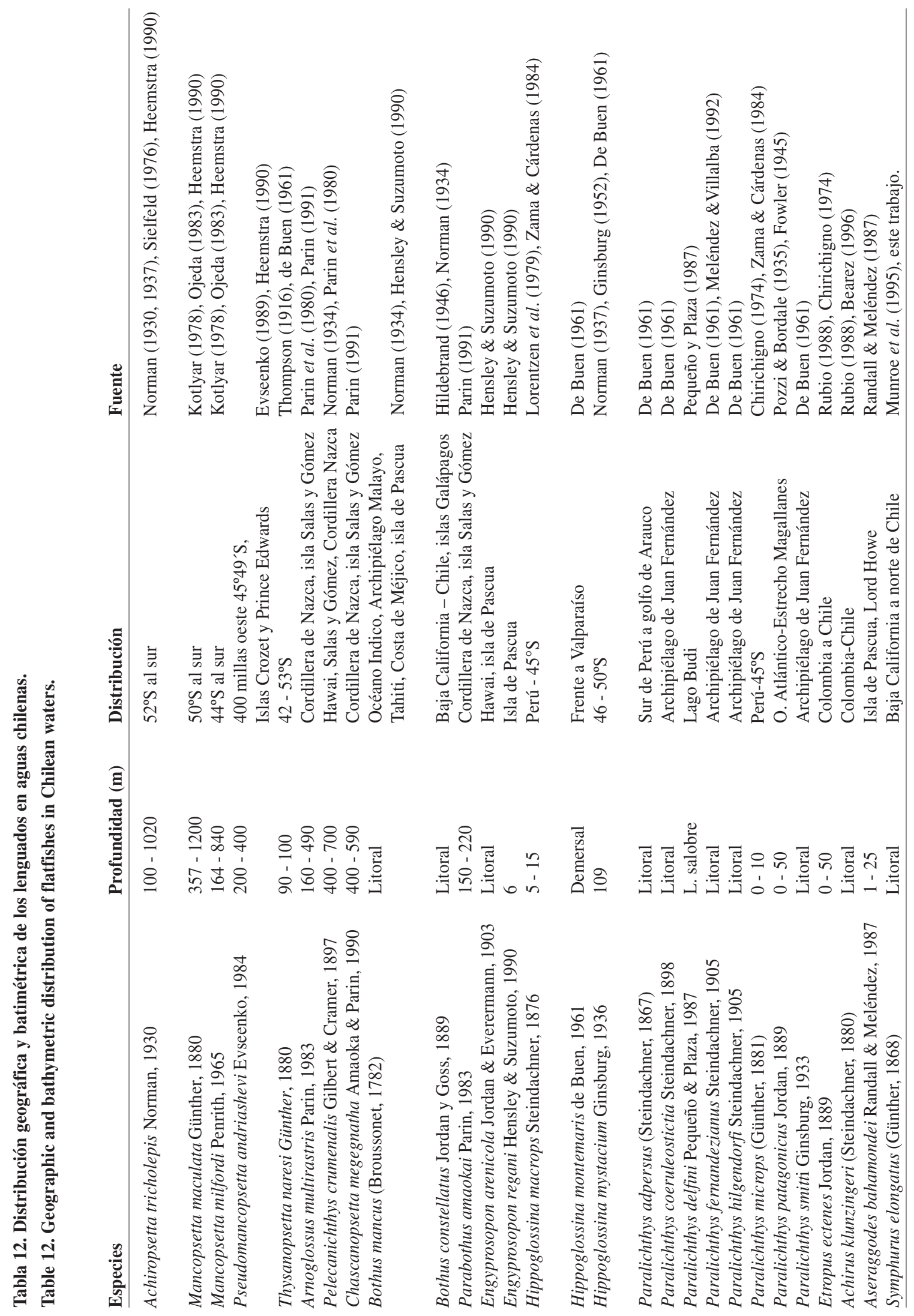


delfini) (Pequeño \& Plaza 1987) y alcanzan hasta Magallanes (Hippoglossina mystacium y Paralichthys patagonicus) (Venegas \& Sielfeld, 1998; Sielfeld \& Vargas, 1999). Las especies de Paralichthys habitan preferentemente el litoral somero (0-50 m) y aquellas de Hippoglossina profundidades mayores (bajo $5 \mathrm{~m}$ y al menos hasta $150 \mathrm{~m}$ ) (Tabla 12).

El litoral de las islas oceánicas (islas de Pascua, Salas y Gómez, San Ambrosio, San Félix) está caracterizado por la presencia de especies de lenguados de tipo indopacífica (Parin, 1991; Pequeño y Lamilla, 1996, 2000) con representantes de la géneros Bothus y Engyprosopon. Los montes submarinos de la cordillera de Nazca son habitados por los géneros Arnoglossus, Parabothus, Pelecanichthys y Chasconopsetta, y que frecuentan aguas mas profundas ( $>150 \mathrm{~m}$ ) (Tabla 12).

El litoral austral o magallánico se caracteriza por la presencia de especies de la familia Archiropsettidae (géneros Achiropsetta, Mancopsetta y Pseudomancopsetta) la mayoría también presentes en el Atlántico sur (Nakamura, 1986; Venegas \& Sielfeld, 1998).

Finalmente, y tal como han señalado Balech (1954), Pequeño \& Lamilla (1993) y Sielfeld \& Vargas (1996) la ictiofauna nerítica del litoral del extremo norte de Chile tiene características de transición entre el conjunto de especies centro-chilenas, también denominado "grupo cálido-temperado de peces" por Ojeda et al. (2000) y el conjunto panameño-peruano. Sus límites están sujetos a los cambios faunísticos que derivan de los eventos tipo ENSO, que entre otros también afectan a los lenguados de las familias Cynoglossidae (género Symphurus) y Achiridae (género Achirus).

El límite entre ambos conjuntos faunísticos presenta fluctuaciones, que en el caso de los peces analizados por Sielfeld et al. (2002), derivan entre otros factores, de cambios faunísticos asociados a eventos tipo ENSO. Durante estos, se habría verificado en el caso de los lenguados, el ingreso a la zona norte de Chile de las familias Cynoglossidae (género Symphurus) y Achiridae (género Achirus), ambas de litoral somero (Tabla 12).

\section{REFERENCIAS}

Ahlstrom, E.H., K. Amaoka, D.A. Hensley, H.G. Moser \& B.Y. Sumida. 1984. Pleuronectiformes: development. En: H.G. Moser, W.J. Richards, D.M. Cohen, M.P. Fahay, A.W. Kendall \& S.L. Richardson (eds.). 1984. Ontogeny and systematics of fishes, Amer. Soc. Ichthyol. Herpetol., Spec. Publ. 1: 640-670.

Amaoka, K. 1969. Studies on the sinistral flounders found in the waters around Japan. Taxonomy, anatomy and phylogeny. J. Shimonoseki Univ. Fish., 18(2): 1-276.

Amaoka, K. \& V. Parin. 1990. A new flounder, Chascanopsetta megegnatha, from the Sala $\mathrm{y}$ Gómez submarine ridge, eastern Pacific Ocean (Teleostei: Pleuronectiformes, Bothidae). Copeia, 1990(3): 717-722.

Bahamonde, N. \& G. Pequeño. 1975. Peces de Chile. Lista Sistemática. Mus. Nac. Hist. Nat., Chile. Publ. Ocas., 21: 1-20.

Balech, E. 1954. División zoogeográfica del litoral sudamericano. Rev. Biol. Mar., Valparaíso, 4(1-7): 184-195.

Béarez, P. 1996. Lista de los peces marinos del Ecuador continental. Rev. Biol. Trop., 44(2): 731-741.

Cervigón, F. 1985. Las especies de los géneros Achirus y Trinectes (Pisces: Soleidae) de las costas de Venezuela. Fundación Científica Los Roques, Caracas, Monogr., 2: 1-83.

Chabanaud, P. 1949. Le problème de la phylogenése des Heterosomata. Bull. Inst. Oceanogr., Monaco, 950: 1-24.

Chapleau, F. 1993. Pleuronectiform relationships: a cladistic reassessment. Bull. Mar. Sci., 52(1): 516540.

Chapleau, F. \& A. Keast. 1988. A phylogenetic reassessment of the monophyletic status of the family Soleidae, with notes on the suborder Soleoidei. Can. J. Zool., 66: 2797-2810.

Chirichigno, N. 1974. Clave para identificar los peces marinos del Perú. Inf. Inst. Mar. Perú-Callao, 44: 1-387.

Chirichigno, N. \& J. Vélez. 1998. Clave para identificar los peces marinos del Perú. Instituto del Mar del Perú, Publ. Esp., 496 pp. 
De Buen, F. 1950. El Mar de Solís y su fauna de peces ( $2^{\text {a }}$ Parte). Servicio Oceanográfico y de Pesca (SOYP). Publ. Científicas, 2: 1-143.

De Buen, F. 1959. Lampreas, tiburones, rayas y peces en la Estación de Biología Marina de Montemar, Chile. Rev. Biol. Mar., Valparaíso, 9(1-3): 3-200.

De Buen, F. 1961. Peces chilenos. Familias Alepocephalidae, Muraenidae, Sciaenidae, Scorpaenidae, Liparidae y Bothidae. Rev. Biol. Mar., Valparaíso, 11(1): 1-52.

Delfín, F.T. 1899. Catálogo de los peces de Chile. Rev. Chil. Hist. Nat., 3: 24, 57-62, 79-85, 95-99, 110113, 121-124, 131-134, 153-161.

Delfín, F.T. 1900. Catálogo de los peces de Chile. Rev. Chil. Hist. Nat., 4:4-6, 21-25, 29-31, 55-58, 6971, 82-84, 110-111, 129-131, 166-167, 185-186.

Evseenko, S.A. 1984. A new genus and species of the armless flounder Pseudomancopsetta andriashevi gen. sp. nov. (Pleuronectiformes) and their position within the Suborder Pleuronectoidei. Vopr. Ikhthiol., 24(5): 709-717.

Evseenko, S.A. 1987. Review of the armless flounder genus Achiropsetta (Pleuronectoidei) with description of a new species Achiropsetta heterolepis sp. nov. Vopr. Ikhtiol., 27(5): 771-783.

Evseenko, S.A. 1989. New data on the armless flounder Pseudomancopsetta andriashevi (Achiropsettidae) from the Southern Ocean. Vopr. Ikhtiol., 29(2): 326-328.

Evseenko, S.A. 1990. On the taxonomic status of the armless flounder Achiropsetta slavae Andriashev (Achiropsettidae). Vopr. Ikhtiol., 30(5): 773-783.

Evseenko, S.A. 1996. Ontogeny and relationships of the flatfishes of the Southern Ocean (Achiropsettidae, Pleuronectoidei). J. Ichthyol., 36(9): 687-712.

Evseenko, S.A. 1997. Revision of the flounder genus Mancopsetta Gill, 1881 (Achiropsettidae, Pleuronectiformes) from the Southern Ocean. J. Ichthyol., 37(6): 411-430.

Evseenko, S.A. 1998. Morphology and identification of two closely related flatfishes of the genus Paralichthys (Paralichthyidae, Pleuronectiformes) from Chilean waters. J. Ichthyol., 38(9): 804-808.
Fowler, H.W. 1945. Fishes of Chile, Systematic Catalog. Parts I and II. Rev. Chil. Hist. Nat., 36: 1171.

Ginsburg, I. 1952. Flounders of the genus Paralichthys and related genera in American waters. U.S. Fish. Bull., 52(71): 1-51.

Heemstra, P.C. 1990. Fam. Achiropsettidae. En: O. Gon. \& P.C. Heemstra (eds.). Fishes of the Southern Ocean. J.L.B. Smith Institute of Ichthyology, pp. 408-413.

Hensley, D.A. \& E. Ahlstrom. 1984. Pleuronectiformes: relationships. En: H.G. Moser, W.J. Richards, D.M. Cohen, M.P. Fahay, A.W. Kendall \& S.L. Richardson (eds.). Ontogeny and systematics of fishes. Amer. Soc. Ichthyol. Herp., Spec. Publ. 1: 670-687.

Hensley, D.A. \& A.Y. Suzumoto. 1990. Bothids of Easter Island, with description of a new species of Engyprosopon (Teleostei: Pleuronectiformes). Copeia, 1990(1): 130-137.

Hildebrand, S.F. 1946. A descriptive catalog of the shore fishes of Perú. U.S. Nat. Mus. Bull., 189: 1530 .

Hubbs, C.L. 1945. Phylogenetic position of the Citharidae, a family of flatfishes. Misc. Publ. Mus. Zool. Univ. Michigan, 63: 1-38.

Hubbs, C. \& K.F. Lagler. 1958. Fishes of the Great Lake Region. Bull. Cranbrook Inst. Sci., 26: 1-213.

Kong, I., J. Tomicic \& J. Zegers. 1985. Ictiofauna asociada al fenómeno El Niño 1982-83 en la zona norte de Chile. Invest. Pesq., Chile, 32: 215-224.

Krupp, F. 1995. Fam. Achiridae. En: W. Fischer, F. Krupp, W. Schneider, C. Sommer, K.E. Carpenter \& V.H. Niem (eds.). Guía FAO para la identificación de especies para los fines de la pesca. Pacífico Centro-Oriental, 2: 845-850.

Kotlyar, A.N. 1978. A contribution to the systematics of "armless" flounders (Pisces, Bothidae) from the South-Western Atlantic. J. Ichthyol., 18(5): 708721.

Li, S.Z. 1981. On the origin, phylogeny and geographical distribution of the flatfishes (Pleuronectiformes). Trans. Chin. Ichthyol. Soc., 1981(1): 11-20. 
Lorentzen, S., C. Gallardo \& J. Jara. 1979. Mariscos y peces de importancia comercial en el sur de Chile. Universidad Austral de Chile, 131 pp.

Mann, G. 1954. La vida de los peces en aguas chilenas. Instituto de Investigaciones Veterinarias y Universidad de Chile. Santiago, Chile. 343 pp.

Massay, S. 1983. Revisión de la lista de peces marinos del Ecuador. Bol. Cient., Inst. Pesca, Ecuador, 6(1): 1-112.

Meléndez, R. \& C. Villalba. 1992. Nuevos registros y antecedentes para la ictiofauna del archipiélago de Juan Fernández. Estud. Oceanol., 11: 3-29.

Munroe, T.A. 1992. Interdigitation patterns of dorsal-fin pterygophores and neural spines, an important diagnostic character for symphurine tonguefishes (Symphurus, Cynoglossidae: Pleuronectiformes). Bull. Mar. Sci., 50: 357-403.

Munroe, T.A., A. Brito \& C. Hernández. 2000. Symphurus insularis: A new Eastern Atlantic dwarf tonguefish (Cynoglossidae: Pleuronectiformes). Copeia, 2000(2): 491-500.

Munroe, T.A., F. Krupp \& M. Schneider. 1995. Fam. Cynoglossidae. En: W.Fischer, F. Krupp, W. Schneider, C. Sommer, K.E. Carpenter \& V.H. Niem (eds.). Guía FAO para la identificación de especies para los fines de la pesca. Pacífico Centro-Oriental, 2: 1039-1059.

Nakamura, I. 1986. Important fishes trawled off Patagonia. En: I. Nakamura (ed.). Japan Marine Fishery Resource Research Center, Tokyo, 365 pp.

Nelson, J. 1994. Fishes of the world. John Wiley \& Sons, New York, 600 pp.

Norman, J.R. 1930. Oceanic fishes and flatfishes collected in 1925-1927. Discovery Rep., 2: 261370.

Norman, J.R. 1934. A systematic monograph of the flatfishes (Heterosomata): Psettodidae, Bothidae, Pleuronectidae. Brit. Mus., London, 459 pp.

Norman, J.R. 1937. Coast fishes. Part II. The Patagonian Region. Discovery Rep., 16: 1-150.

Ojeda, F.P. 1978. Apterygopectus avilesi n. sp. nuevo género y nueva especie de lenguado para aguas australes chilenas. Mus. Nac. Hist. Nat, Chile, Not. Mensual, 23(267): 3-10.
Ojeda, F.P. 1983. Distribución latitudinal y batimétrica de la ictiofauna demersal del extremo austral de Chile. Rev. Chil. Hist. Nat., 56: 61-70.

Ojeda, F.P., F.A. Labra \& A.A. Muñoz. 2000. Biogeographic patterns of Chilean littoral fishes. Rev. Chil. Hist. Nat., 73: 625-641.

Paiva Carvalho, J., L.H. Tommasi \& M.D. Novelli. 1968. Lista dos linguados do Brasil. Contr. Inst. Oceanogr. Univ. S. Paulo, ser. Ocean. Biol., 14: 128.

Parin, N.V. 1983. Two new species of bothid flatfishes (Bothidae, Pleuronectiformes) from the Nazca submarine ridge. Bull. Moskovskogo Obshch. Ispyt. Prirody, 88(4): 90-96.

Parin, N.V. 1991. Fish fauna of the Nazca and Sala y Gomez submarine ridges, the easternmost outpost of the indo-west Pacific zoogeographic region. Bull. Mar. Sci., 49(3): 671-683.

Parin, N.V., G.A. Golovan, N.P. Pakhorukov, Y.I. Sazonov \& Y.N. Shcherbachev. 1980. Fishes from the underwater Nazca and Sala y Gomez ridges collected in the cruise of the R/V "Ichthyandr". pp. 5-18. En: N.V. Parin (ed.). Fishes of open ocean. P.P. Shirshov Institute of Oceanology, Moscow, 120 pp.

Pequeño, G. 1989. Peces de Chile. Lista sistemática revisada y comentada. Rev. Biol. Mar., Valparaíso, 24(2): 1-132.

Pequeño, G. 1997. Peces de Chile. Lista sistemática revisada y comentada: addendum. Rev. Biol. Mar. Oceanogr., 32(2):77-94.

Pequeño, G. \& J. Lamilla. 1993. Batoideos comunes a las costas de Chile y Argentina-Uruguay (Pisces: Chondrichthyes). Rev. Biol. Mar., Valparaíso, 28(2): 203-217.

Pequeño, G. \& J. Lamilla. 1996. Desventuradas Islands, Chile: the easternmost outpost of the IndoWest Pacific Zoogeographic Region. Rev. Biol. Trop., 44(2): 929-931.

Pequeño, G. \& J. Lamilla. 2000. The littoral fish assemblage of the Desventuradas Islands (Chile) has zoogeographical affinities with the Western Pacific. Global Ecol. Biogeogr., 9: 431-437.

Pequeño, G. \& R. Plaza. 1987. Descripción de Paralichthys delfini n. sp., con notas sobre otros lenguados congenéricos de Chile (Pleuronectifor- 
mes, Bothidae). Rev. Biol. Mar., Valparaíso, 23(7): 159-172.

Pozzi, A \& L. Bordale. 1935. Cuadro sistemático de los peces marinos de la República Argentina. Ans. Soc. Cient. Arg., 120: 1-47.

Randall, J.E. \& R. Meléndez. 1987. A new sole of the genus Aseraggodes from Easter Island and Lord Howe Island, with comments on the validity of $A$. ramsaii. Bishop Mus. Occas. Pap., 27: 97-105.

Rubio, E.A. 1988. Peces de importancia comercial para el Pacífico Colombiano. Universidad del Valle, Departamento de Biología, Cali, 495 pp.

Sielfeld, W. 1976. Presencia del género Achiropsetta Norman, 1930 (Pisces, Pleuronectiformes) en aguas patagónicas chilenas. Ans. Inst. Pat. Punta Arenas, 7: 215-217.

Sielfeld, W. \& M. Vargas. 1996. Composición y estructura de la ictiofauna demersal en la zona norte de Chile. Invest. Mar., Valparaíso, 24: 3-17.

Recibido: 14 junio 2002; Aceptado: 15 abril 2003
Sielfeld, W. \& M. Vargas. 1999. Review of marine fish zoogeography of Chilean Patagonia $\left(42^{\circ}-57^{\circ} \mathrm{S}\right)$. Sci. Mar., 63(Supl. 1): 451-463.

Steindachner, F. 1905. Die Fische der Sammlung Plate. Fauna Chilensis L. Plate. III: 201 - 214.

Thompson, W.F. 1916. Fishes collected by the U.S. Bureau of Fisheries Steamer "Albatross" during 1888 between Montevideo, Uruguay and Tomé, Chile on the voyage through the Straits of Magellan. Proc. U.S. Nat. Mus., 50: 401-476.

Venegas, C. \& W. Sielfeld. 1998. Catálogo de los vertebrados de la región de Magallanes y Antártica Chilena. Ediciones Univ. Magallanes, Punta Arenas, $122 \mathrm{pp}$.

Zama, A. \& E. Cárdenas. 1984. Descriptive catalogue of marine and freshwater fishes from the Aysen Region, Southern Chile, with zoogeographical notes on the fish fauna. Servidcio Nacional de Pesca, Chile-Japan International Cooperation Agency (JICA), 75 pp. 